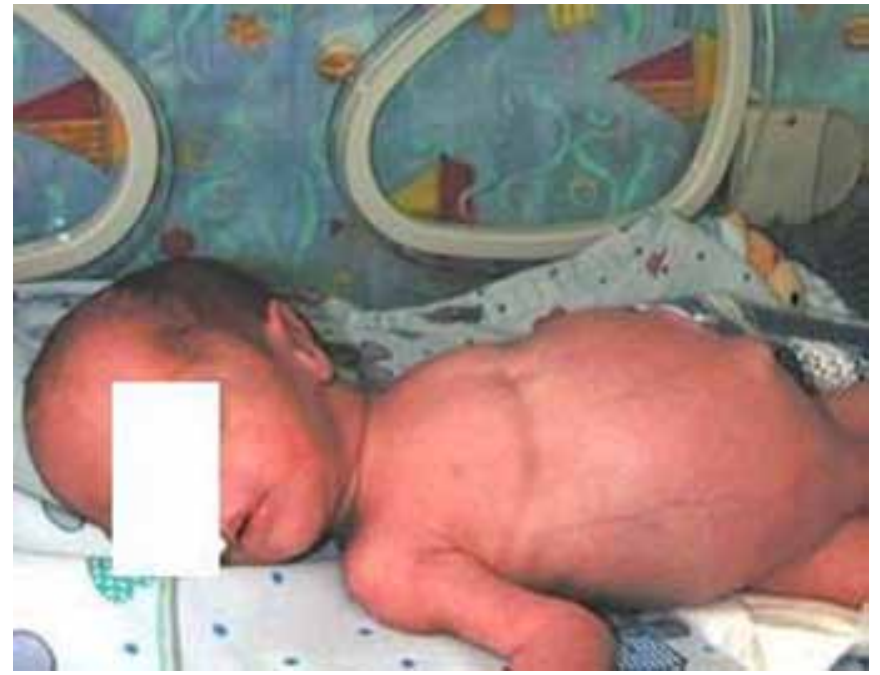

Abstract 552 Figure 1 Abdominal distention

Feeds were stopped. Investigations ruled out NEC. After 2 days the infant improved clinically and feeding was recommenced. A further episode occurred, coinciding with reintroduction of full expressed breast milk feeds. Further maternal dietary history revealed an abnormally high intake of uncooked onions. After removing onion from her diet the problem resolved.

Conclusions Onion is used in complementary medicine for antimicrobial, antifungal, glucose and lipid lowering properties. Human studies have associated high maternal intake with infantile colic in breastfeeding infants. The intestinal flora of premature infants is immature, hindering gut absorption and metabolism. Abdominal distension occurs as gas builds up in the bowel.

A diet containing plentiful fruit and vegetables is advocated for breastfeeding mothers. In the case of onion and cruciferous vegetables awareness of the potential effect on the immature gut is important.

\section{DISPARITIES IN COGNITIVE DOMAINS SEEN IN PATIENTS WITH KABUKI SYNDROME}

doi:10.1136/archdischild-2012-302724.0553

${ }^{1} \mathrm{M}$ Nakamura, ${ }^{1} \mathrm{M}$ Hirai, ${ }^{1} Y$ Muramatsu, ${ }^{2} \mathrm{~S}$ Mizuno, ${ }^{2} \mathrm{~A}$ Matsumoto. 'Institute for Developmental Research, Aichi Human Service Center; ${ }^{2}$ Central Hospital, Aichi Human Service Center, Kasugai, Japan

Background It is important to clarify the characteristic traits of the cognitive functions of Kabuki syndrome patients in order to choose appropriate pedagogical techniques.

Methods The cognitive functions in seven participants with Kabuki syndrome were investigated using the Kaufmann assessment battery for children test, the Benton facial recognition test and Theory of Mind test, with some copying tasks of two and three dimensional line drawing figures. The results were compared to those of seven Williams syndrome participants.

Results The findings indicated disparities among cognitive areas in the Kabuki syndrome participants with stronger subtest "number recall" than the subtest "gestalt closure" in the Kaufmann assessment battery for children test $(\mathrm{p}<0.05)$. The disparities were compatible as previously described. The difficulties in copying the line drawing figures suggested a dorsal pathway dysfunction similar to that in Williams syndrome patients, but further longitudinal observation is needed. In the Kabuki syndrome participants, four of five participants who could perform the Theory of Mind test could pass the test, whereas only two out of six in the Williams syndrome patients could do so. The discrepancies between the results of the Benton facial recognition test and Theory of Mind test were the opposite of those in the Williams syndrome patients, in spite of anecdotal observations of similar tendencies in social interaction.

Conclusion Kabuki syndrome is another disease that shows disparities among cognitive functions. Investigating this syndrome may help us to understand the mechanisms of human cognitive function.

\section{KOOLAN SYNDROME IS A NOVEL GENOMIC DISORDER WITH MENTAL HANDICAP MULTIPLE CONGENITAL ANOMALY DUE TO MICRO DELETION AT 17021.31}

doi:10.1136/archdischild-2012-302724.0554

AM Ismail, MJ Mahony, AM Murphy, W Oreadon. Paediatric Department, Mid Western Regional Hospital University of Limerick, Limerrick, Ireland

Aim Our aim is to report a case of koolan syndrome in a 3 year old male child.

Methods Clinical history, physical examination, clinical photography results of molecular genetic testing are presented.

Results A male infant was born to a healthy Irish Caucasian non consangnious couple by normal vaginal delivery at 38 weeks gestation, Birth weight $2.3 \mathrm{kgs}$, and head circumference $33.5 \mathrm{cms}$. It was his mothers third pregnancy. The first was a molar pregnancy. Second resulted in a birth of an Edward's syndrome who died at 3 weeks of age.

At birth he was hypotonic admitted to neonatal unit with low blood glucose of $1.6 \mathrm{mmol} / \mathrm{L}$ and on examination he has low set ears, long face widely spaced nipples narrow palpebral fissures and right undescended testis with feeding difficulty in neonatal period for which he required nasogastric feeds for first 3 weeks of life. With in last 3 years he is having global developmental delay pleasant behaviour and learning difficulties.

\section{PERINATAL NEUROBLASTOMA WITH A GERMLINE INTERSTITIAL 2P DUPLICATION INVOLVING THE MYCN GENE: A CASE REPORT}

doi:10.1136/archdischild-2012-302724.0555

${ }^{1,2} \mathrm{D}$ Warad, ${ }^{2,3} \mathrm{~S}$ Kirmani, ${ }^{2} \mathrm{P}$ Marri Reddy, ${ }^{2,4} \mathrm{M}$ Nemergut, 1,2V Rodriguez, ${ }^{1,2} \mathrm{~A}$ Nageswara Rao. 'Division of Pediatric Hematology-Oncology; ${ }^{2}$ Department of Pediatric and Adolescent Medicine; ${ }^{3}$ Department of Medical Genetics; ${ }^{4}$ Department of Anesthesiology, Mayo Clinic, Rochester, MN, USA

Background/Aims MYCN proto-oncogene is located on chromosome 2 p24. MYCN amplification is a poor prognostic factor in neuroblastoma. However, the role of germline MYCN copy number gain is unclear. It is unknown if it is a prerequisite for MYCN amplification or an independent event in neuroblastoma.

Methods Case report of perinatal neuroblastoma with a mosaic interstitial $2 \mathrm{p}$ duplication and literature review.

Results A $3.3 \mathrm{~cm}$ right suprarenal mass was noted in a 2 day old infant with bilateral postaxial polydactyly, syndactyly and bicuspid aortic valve. He was observed clinically until 3 weeks of age when he presented with increasing abdominal distension, prominent hepatomegaly, enlarging suprarenal mass, and marked elevation of urinary VMA/HVA levels. Diffuse liver MIBG avidity was noted. Emergent chemotherapy was started and he underwent decompressive laparotomy secondary to abdominal compartment syndrome. He is currently five months into therapy and doing well. aCGH performed on peripheral blood leukocytes showed mosaic interstitial duplication from 2 p24.1 to 2 p25.3 involving the 